\title{
Triptolide abrogates growth of colon cancer and induces cell cycle arrest by inhibiting transcriptional activation of E2F
}

\author{
Amanda R Oliveira' ${ }^{1}$, Georg Beyer ${ }^{1}$, Rohit Chugh, Steven J Skube, Kaustav Majumder, Sulagna Banerjee, \\ Veena Sangwan, Lihua Li, Rajinder K Dawra, Subbaya Subramanian, Ashok K Saluja and Vikas Dudeja
}

Despite significant progress in diagnostics and therapeutics, over 50 thousand patients die from colorectal cancer annually. Hence, there is urgent need for new lines of treatment. Triptolide, a natural compound isolated from the Chinese herb Tripterygium wilfordii, is effective against multiple cancers. We have synthesized a water soluble analog of triptolide, named Minnelide, which is currently in phase I trial against pancreatic cancer. The aims of the current study were to evaluate whether triptolide/Minnelide is effective against colorectal cancer and to elucidate the mechanism by which triptolide induces cell death in colorectal cancer. Efficacy of Minnelide was evaluated in subcutaneous xenograft and liver metastasis model of colorectal cancer. For mechanistic studies, colon cancer cell lines HCT116 and HT29 were treated with triptolide and the effect on viability, caspase activation, annexin positivity, lactate dehydrogenase release, and cell cycle progression was evaluated. Effect of triptolide on E2F transcriptional activity, mRNA levels of E2F-dependent genes, E2F1- retinoblastoma protein (Rb) binding, and proteins levels of regulator of G1-S transition was also measured. DNA binding of E2F1 was evaluated by chromatin immunoprecipitation assay. Triptolide decreased colon cancer cell viability in a dose- and time-dependent fashion. Minnelide markedly inhibited the growth of colon cancer in the xenograft and liver metastasis model of colon cancer and more than doubles the median survival of animals with liver metastases from colon cancer. Mechanistically, we demonstrate that at low concentrations triptolide induces apoptotic cell death but at higher concentrations it induces cell cycle arrest. Our data suggest that triptolide is able to induce G1 cell cycle arrest by inhibiting transcriptional activation of E2F1. Our data also show that triptolide downregulates E2F activity by potentially modulating events downstream of DNA binding. Therefore, we conclude that Triptolide and Minnelide are effective against colon cancer in multiple pre-clinical models.

Laboratory Investigation (2015) 95, 648-659; doi:10.1038/labinvest.2015.46; published online 20 April 2015

Colorectal cancer is the third most common cause of cancerrelated deaths in the United States. ${ }^{1}$ Despite significant progress in the prevention, early diagnosis and management of colorectal cancer, over 50 thousand patients died from this disease in 2012. ${ }^{1}$ Oxaliplatin is the latest addition to the armamentarium against colon cancer and its addition to the original adjuvant therapy regimen of 5-FU and leucovorin improves the 5-year disease-free survival of stage II and III patients from $67 \%$ to $73 \%$ with minimal improvement in overall survival. ${ }^{2}$ Those presenting with metastatic disease have a grim prognosis with median survival ranging from 15 to 20 months. ${ }^{3}$ Thus, there is an urgent need to develop effective therapies for this formidable disease.
Triptolide, a natural compound isolated from the Chinese plant Tripterygium wilfordii, has anti-inflammatory and anticancer properties. Previously, we and others have shown that triptolide is effective against multiple types of cancer. Triptolide induces cell death in pancreatic cancer cells and markedly reduces the growth and locoregional spread of pancreatic tumors in animal models. ${ }^{4}$ We have also shown that triptolide is effective against osteosarcoma, ${ }^{5}$ lung cancer, ${ }^{6}$ cholangiocarcinoma, ${ }^{7}$ and neuroblastoma. ${ }^{8}$ Others have shown that triptolide is effective against melanoma, ${ }^{9}$ gastric, ${ }^{9}$ breast, ${ }^{9}$ and colon cancer. ${ }^{10,11}$ We have now synthesized the water soluble analog of triptolide, named Minnelide, and have evaluated it extensively in multiple

Division of Basic and Translational Research, Department of Surgery, University of Minnesota, Minneapolis, MN, USA

Correspondence: Dr V Dudeja, MBBS, Division of Basic and Translational Research, Department of Surgery, University of Minnesota, Minneapolis, MN 55455, USA. E-mail: dudej001@umn.edu

${ }^{1}$ These authors contributed equally to this work.

Received 19 August 2014; revised 18 February 2015; accepted 27 February 2015 
animal models and scenarios of pancreatic cancer ${ }^{12}$ with encouraging results. Whether Minnelide is efficacious against colon cancer is not known.

Although our previous studies suggest that the downregulation of $\mathrm{HSP} 70,{ }^{4} \mathrm{Mcl}-1,{ }^{13}$ and $\mathrm{Sp} 1^{14}$ contribute to triptolide-induced cancer cell death, the exact mechanism of action of triptolide remains elusive. Our previous work also suggests that triptolide can induce multiple types of programmed cell death, inducing apoptosis and autophagy depending on the cancer cell type. ${ }^{15}$ In the current study we demonstrate that Minnelide is effective against colon cancer in the xenograft and liver metastasis model. Mechanistically, we show that at low concentrations triptolide induces cell death by apoptosis, but at higher concentrations it induces cell cycle arrest. Furthermore, our studies reveal that triptolide is able to induce G1 cell cycle arrest by inhibiting transcriptional activation of E2F. Our data also suggest that triptolide downregulates E2F activity by potentially modulating events downstream of its DNA binding.

\section{MATERIALS AND METHODS Reagents}

Colorectal cancer cell lines HCT116 and HT29 were purchased from ATCC (Manassas, VA, USA). Human colon epithelial cells (HCEC) were a generous gift from Professor Shay. ${ }^{16}$ Triptolide, Guava Nexin Apoptosis kit, Guava cell cycle reagent, phosphatase inhibitor cocktail II were purchased from EMD Millipore Chemicals (Billerica, MA, USA). DMSO was purchased from Sigma Aldrich (St Louis, MO, USA). Cell Counting Kit-8 was from Dojindo Molecular Technologies (Rockville, MD, USA). FuGENE-HD transfection reagent, Dual-Luciferase Assay system, Caspase-Glo 3/7 assay and CytoTox 96 Non-Radioactive Cytotoxicity Assay were from Promega (Madison, WI, USA). QuantiTect SYBR Green PCR kit was from Qiagen (Valencia, CA, USA). Cignal E2F Reporter kit was from SABiosciences, Qiagen. TRIzol Reagent was from Life Technologies (Great Island, NY, USA). Phosphate-buffered radio immunoprecipitation assay (RIPA) buffer, beta-glycerophosphate and sodium-pyrophosphate were from Boston BioProducts (Ashland, MA, USA). Protease inhibitors were from Roche (Mannheim, Germany). TrisHCL polyacrylamide gels and nitrocellulose membranes were from BioRad laboratories (Richmond, CA, USA). BCA protein assay kit was from Pierce (Rockford, IL, USA). High Capacity cDNA Reverse Transcription Kit was from Applied Biosystems (Foster City, CA, USA). McCoy's 5A medium was from Thermo Scientific (South Logan, UT, USA). For western blotting antibodies against cyclin-dependent kinase 4 (CDK-4), cyclin-dependent kinase 6 (CDK-6), p15, and cyclins A, D1, and E2 were purchased from Cell Signaling (Danvers, MA, USA). All secondary antibodies as well as streptavidin HRP conjugate used in the study were also purchased from Cell Signaling. Biotin-conjugated $\mathrm{Rb}$ antibody was from Thermo Scientific. Antibody against p21 and biotin-conjugated antibody against E2F1 were from Abcam (Cambridge,
MA, USA). Beta-Actin primary antibody was purchased from Santa Cruz Biotechnologies (Santa Cruz, CA, USA).

\section{Cell Culture and Drug Treatment}

Colorectal cancer cell lines HCT116 and HT29 were cultured in McCoy's 5A medium supplemented with 10\% FBS and 1\% penicillin-streptomycin at $37^{\circ} \mathrm{C}$ in a humidified $5 \% \mathrm{CO}_{2}$ atmosphere as recommended by ATCC. The immortalized epithelial cell HCEC were maintained with DMEM media (HyClone, Logan, UT, USA) supplemented with EGF ( $25 \mathrm{ng} / \mathrm{ml}$, Cell Signaling), hydrocortisone ( $1 \mu \mathrm{g} / \mathrm{ml}$, Sigma), insulin $(10 \mu \mathrm{g} / \mathrm{ml})$, transferrin $(2 \mu \mathrm{g} / \mathrm{ml})$, sodium selenite (5 nм) (Gibco), 2\% cosmic calf serum (HyClone), gentamicin (Gibco) and amphotericin B (Corning) in Primaria flasks (BD Biosciences) under $2 \%$ oxygen, $5 \%$ carbon dioxide and $93 \%$ nitrogen. Triptolide was dissolved in DMSO to provide a stock solution of $1 \mathrm{mg} / \mathrm{ml}$. Cells were treated with triptolide at final concentrations of 0-200 nm in McCoy medium with 5\% FBS and $1 \%$ penicillin/streptomycin antibiotics.

\section{Determination of Cell Viability}

Cell viability was determined using the Dojindo Cell Counting Kit-8. After treatment with triptolide for the appropriate duration $(24-72 \mathrm{~h})$, cell viability was measured by incubation with $10 \mu \mathrm{l}$ of the tetrazolium substrate for $1 \mathrm{~h}$ at $37^{\circ} \mathrm{C}$; absorbance at $450 \mathrm{~nm}$ was measured.

\section{Animal Experiments}

All animal experiments were reviewed and ratified by the Institutional Animal Care and Use Committee at the University of Minnesota. Three different animal models were carried out. For the xenograft model $1 \times 10^{6}$ HCT116 cells were suspended in matrigel and injected subcutaneously in the right flank of 2-month-old female nude mice. Seven days post implantation, the mice were randomized into following two groups: (1) control (injected with saline only) and (2) Minnelide $(0.42 \mathrm{mg} / \mathrm{kg} /$ day $)$. Mice were treated daily and tumor size was measured weekly and tumor volume was calculated as $0.52 \times$ length $\times$ width $^{2}$. This model was terminated after 30 days.

For the liver metastasis model, animals were anesthetized with a mixture of ketamine/xylazine $(100 \mathrm{mg} / \mathrm{kg}$ and $10 \mathrm{mg} /$ $\mathrm{kg}) .1 \times 10^{6}$ HCT116 cancer cells suspended in matrigel were injected under the splenic capsule at laparotomy and wound was closed using absorbable sutures and surgical staples. Five days post injection mice were randomized into control (injected with saline only) and Minnelide $(0.42 \mathrm{mg} / \mathrm{kg} /$ day $)$ groups. Four weeks after the start of the treatment animals were euthanized and liver metastases were dissected out. The number and cumulative weight of visible liver metastases was compared between two groups.

In a separate liver metastasis model, animal survival was used as the end point. In this model $2 \times 10^{6}$ cells were injected under spleen capsule and liver tumors were allowed to develop. Four weeks post implantation, animals were 
randomized and treated as described above. In accordance with animal care guidelines, animals were euthanized if they lost $>20 \%$ of baseline weight, developed excessive ascites, or showed any other signs of failure to thrive. This was considered death of the animal. The experimentation was terminated 80 days after start of treatment.

\section{Caspase-3 Activity Assay}

Caspase- 3 activity was measured by the Caspase-Glo 3/7 assay from Promega according to the manufacturer's protocol.

\section{Measurement of Annexin-V-Positive Cells}

For evaluation of phosphatidylserine externalization (marker of early apoptosis), after appropriate duration of treatment with triptolide, colon cancer cells were stained with Guava Nexin reagent according to the manufacturer's protocol and then analyzed (5000 events) on a Guava PCA flow cytometer.

\section{LDH Release Assay}

Necrosis was measured by lactate dehydrogenase (LDH) release assay. In brief, after triptolide treatment, LDH activity in the supernatant and in the whole cell pellet (after lysing the cells) was measured using CytoTox 96 Non-Radioactive Cytotoxicity Assay from Promega according to the manufacturer's protocol. LDH activity in the supernatant when compared with total LDH content provided the percent LDH release and thus the extent of necrosis.

\section{Cell Cycle Analysis}

For cell cycle analysis colon cancer cells were serum starved for $12 \mathrm{~h}$ for cell cycle synchronization and then treated with triptolide (0-200 nM) for 12-24 h. After appropriate treatment cells were harvested by trypsinization, washed with PBS, and resuspended in PBS at a concentration of $10^{6} \mathrm{cells} / \mathrm{ml}$. A $100 \mu \mathrm{l}$ aliquot of this suspension was taken and mixed with $100 \mu \mathrm{l}$ Guava cell cycle reagent, incubated in the dark for 30 min and then acquired on BD flow cytometer.

\section{Luciferase Reporter Assay for E2F}

E2F activity assay was performed using the Cignal reporter assay kit (Cat no. CCS-003L, SABiosciences). In brief, cells were transfected with reporter plasmid (200 ng/well) using FuGENE transfection reagent. Appropriate negative control was used in all experiments. Cells were allowed to grow for $24 \mathrm{~h}$ before triptolide treatment for $12 \mathrm{~h}$. Cells were collected after treatment and analyzed using the Dual-Luciferase reporter assay system (Promega) according to the manufacturer's protocol.

\section{Quantitative Real-time PCR}

Quantitative real-time PCR for Orc1, Cyclin A-1, and CDC-2 was carried out using primers obtained from Qiagen (QuantiTect primer assay). RNA was isolated from the different cell lines and from the tumor samples according to the manufacturer's instructions using TRIzol (Invitrogen).
Total RNA $(1 \mu \mathrm{g})$ was transcribed into cDNA and real-time PCR was performed using the QuantiTect SYBR Green PCR kit (Qiagen) according to the manufacturer's instructions on an Applied Biosystems 7300 real-time PCR system. All data were normalized to the housekeeping 18-S gene (18S QuantiTect primer assay, Qiagen).

\section{Western Blotting}

Protein concentration in cell lysates was estimated using the BCA protein estimation assay (Thermo Scientific). Lysates were western blotted for Cyclin D1, Cyclin E2, CDK-4, CDK-6, p15, p21, Rb, E2F1, and actin using the western blotting protocol described before. ${ }^{17}$

\section{Immunoprecipitation}

For immunoprecipitation $250-500 \mu \mathrm{g}$ of appropriately treated cell lysates were adjusted to a final volume of $500 \mu \mathrm{l}$ and precleared with $1 \mu \mathrm{g}$ of normal rabbit IgG (Santa Cruz Biotechnologies cat\# 2027), and $20 \mu \mathrm{l}$ of nProtein A Sepharose 4 fast flow (GE Healthcare, cat $\# 17-5280-01$ ) at $4^{\circ} \mathrm{C}$ for $30 \mathrm{~min}$ on rotating platform. Following incubation lysates were centrifuged at $1000 \mathrm{~g}$ for 30 seconds at $4^{\circ} \mathrm{C}$, and supernatants were collected and incubated with $1 \mu \mathrm{g}$ of antiE2F1 antibody (Invitrogen cat\#321400), or $1 \mu \mathrm{g}$ of anti-Rb antibody (BD Biosciences cat\#554162) on a rotating platform at $4{ }^{\circ} \mathrm{C}$ overnight. After overnight incubation, $20 \mu \mathrm{l}$ of nProtein A Sepharose 4 fast flow was added to lysates and incubated for $2 \mathrm{~h}$ at $4{ }^{\circ} \mathrm{C}$ with constant rotation. Lysates were then centrifuged at $1000 \mathrm{G}$ for $30 \mathrm{~s}$ at $4{ }^{\circ} \mathrm{C}$ and supernatant was discarded. Sepharose pellets were gently washed four times with $1.0 \mathrm{ml}$ RIPA, and after the final wash pellets were resuspended in $40 \mu \mathrm{l}$ of $(2 \times)$ Laemmli Sample Buffer (BIORAD, cat\#: 161-0737) and boiled for 3 min. Immunoprecipitates were then western blotted and probed for $\mathrm{Rb}$ or E2F1.

\section{Chromatin Immunoprecipitation}

In all, $2 \times 10^{6}$ HCT116 and HT29 cells were plated in $10-\mathrm{cm}$ dishes. Twenty four hours after plating, the cells were treated with triptolide 50, 100, and $200 \mathrm{~nm}$ for 12 hours. Following treatment chromatin was cross-linked, isolated, immunoprecipitated, cleaned, and PCR performed using Pierce Agarose CHIP kit (Cat \# 26156) and using manufacturer's protocol. E2F1 CHIP grade antibody and target CDC-2 primer were obtained from Millipore (Cat \#17-10061).

\section{Statistical analysis}

Values are expressed as mean \pm s.e. All experiments with cells were repeated at least three times. The significance of the difference between the control and each experimental test condition was analyzed by unpaired Student's $t$-test. $\mathrm{P}<0.05$ was considered statistically significant. Survival was analyzed by Kaplan-Meier curves and log-rank test. 


\section{RESULTS}

\section{Triptolide and Minnelide Are Effective against Colon} Cancer In Vitro and in Multiple Mouse Models

The effect of different doses of triptolide on the viability of colon cancer cells was evaluated. As seen in Figures 1a and b, triptolide decreases the viability of HCT116 and HT29 colon cancer cells in a dose- and time-dependent fashion. To evaluate the effect of triptolide on colon cancer in vivo, three different models of colon cancer were employed. The first was a xenograft model where $1 \times 10^{6}$ HCT116 colon cancer cells were implanted subcutaneously, allowed to grow for a week, and randomized into the control (no treatment) and treatment (Minnelide $0.42 \mathrm{mg} / \mathrm{kg} /$ day) arms. As mentioned previously Minnelide is a water soluble analog of triptolide, which we have shown to be very effective against pancreatic cancer in multiple animal models. ${ }^{12}$ Mice were treated for 30 days and the tumor volume was compared between the two groups on a weekly basis. As per University of Minnesota animal care guidelines the experiment was terminated when the tumor volume exceeded $1 \mathrm{~cm}^{3}$. As seen in Figure $1 \mathrm{c}$, Minnelide treatment reduced the growth of colon cancer xenografts significantly when compared with the vehicle alone group. As liver metastases of colorectal cancer is a significant clinical problem, we used the liver metastasis model of colon cancer. In this model HCT116 colon cancer cells were injected under the splenic capsule and allowed to metastasize to the liver. Five days post implantation, the animals were randomized into control (no treatment) and Minnelide $(0.42 \mathrm{mg} / \mathrm{kg} /$ day $)$ treatment groups. Mice were treated with the designated agent for 4 weeks, killed, and liver metastases dissected out. The number and weight of liver metastases was compared between the two groups. As seen in Figures 1d and e, Minnelide treatment reduced the number and the weight of liver metastases significantly when compared with the control group. Photographs of representative tumor implant in the liver are shown in Figure 1f. The left panel shows the tumorliver interface at $\times 10$ and the right panel shows the tumor at higher magnification of $\times 200$. In another independent experiment, we evaluated the impact of Minnelide treatment on survival in the liver metastasis model of colon cancer. In this model animals were randomized 4 weeks post implantation into control (no treatment) and Minnelide $(0.42 \mathrm{mg} / \mathrm{kg} /$ day) treatment groups. Animals were then treated daily and followed for any signs of failure to thrive and $>20 \%$ weight loss. If these signs were observed the animals were euthanized and this was considered cancer-related death. At the end of the model the median survival in the two groups was compared. Figure 1g demonstrates the Kaplan-Meier curve demonstrating the survival in the two treatment groups. As seen in Figure 1g, Minnelide treatment significantly $(P<0.0001)$ improved the survival of animals with liver metastases. To evaluate if the effects of triptolide are specific to transformed cells, we evaluate the effect of triptolide on the viability of normal colonic epithelial cells. As seen in
Figure 1h, triptolide does not affect the viability of normal human colonic epithelial cells, even at high concentrations.

Triptolide Induces Apoptosis at Low Dose but Causes G1 Phase Arrest at Higher Dose

Once we had established the efficacy of Minnelide in decreasing tumor growth and increasing survival, we proceeded to examine the mechanism by which triptolide decreases growth of colon cancer cells. We have previously shown that triptolide can induce cell death in pancreatic cancer cells by multiple mechanisms, including apoptosis and autophagy. ${ }^{15}$ To evaluate the effect of triptolide on apoptotic cell death in colon cancer cells, the effect of triptolide on Annexin-V (a marker of early apoptosis) and caspase-3 (effector caspase of caspase-dependent apoptosis) was measured. As seen in Figures $2 \mathrm{a}-\mathrm{d}$, triptolide treatment results in an increase in annexin $\mathrm{V}$ positivity and caspase- 3 activation in both HCT116 and HT29 colon cancer cells. Interestingly, contrary to previously published data using other cancer cell lines, we did not observe a dose-dependent increase in apoptosis. The caspase- 3 activation and annexin positivity increased up to a triptolide dose of $25-50 \mathrm{~nm}$ and then decreased at concentrations above $50 \mathrm{~nm}$. As we have previously observed that triptolide treatment can lead to programmed cell death by multiple different mechanisms, we evaluated whether triptolide at higher concentrations can induce other forms of cell death in addition to apoptosis. As seen in Figures 2e and $\mathrm{f}$, triptolide treatment does not induce significant necrosis as measured by $\mathrm{LDH}$ release assay. We also evaluated the effect of triptolide on autophagy by measuring LC3 II levels by western blot and did not observe any change (data not shown).

In search for the mechanism by which triptolide induces increased cell death despite reduced apoptosis at higher dosage, we next looked at the effect of triptolide on cell cycle progression in colon cancer cells. As seen in Figures $3 a$ and b, triptolide treatment significantly increases the percentage of cells in G1 phase in both colon cancer cell lines tested as compared with control (from 50\% in untreated cells to $80 \%$ at $200 \mathrm{~nm}$ ). This suggests that at higher concentrations, triptolide induces cell cycle arrest in colon cancer cells. Data from a representative experiment are shown in Figure 3c.

\section{Triptolide Inhibits Transcriptional Activity of E2F, the Key Regulator of G1-S Transition}

We next focused on the mechanism by which triptolide induces G1 cell cycle arrest in colon cancer cells. Activity of E2F transcription factors (E2F1-3) is crucial to progression of cell cycle from G1 to S phase. Given that triptolide treatment induces a G1 cell cycle arrest, we evaluated the effect of triptolide on E2F activity using a luciferase promoter assay. As seen in Figure 4a, triptolide treatment results in a dosedependent decrease in the transcriptional activity of E2F in HCT116 cell line. As E2F1 is the most well understood and characterized member of E2F family of proteins, we focused 
primarily on this subtype. To further confirm that triptolide inhibits transcriptional activity of E2F1, we evaluated the effect of triptolide on downstream targets of E2F1, namely cyclin A, Orc1, and CDC-2. As seen in Figures 4b-d, triptolide treatment leads to a dose-dependent decrease in the expression of cyclin A, Orc1, and CDC-2 in both HCT116 and HT29 cell lines, confirming that E2F1 activity is downregulated by triptolide.

Next, we sought to define the mechanism by which triptolide downregulates the transcriptional activity of E2F1. Phosphorylation of $\mathrm{Rb}$ in E2F1-binding pocket by CDK-4 and CDK-6 in conjunction with cyclin D and CDK-2 in conjugation with cyclin E leads to the release of E2F1, which in turn, can regulate $\mathrm{E} 2 \mathrm{~F} 1$-dependent genes, and thus regulate the G1 to $S$ phase transition. Activity of CDKs is further downregulated by $\mathrm{p} 15, \mathrm{p} 16$, and $\mathrm{p} 21$. We therefore examined the effect of triptolide treatment on the levels of these proteins. We hypothesized that if triptolide-mediated G1 arrest was through its effect on the levels of these proteins, triptolide treatment would result in decreased levels of cyclin D, cyclin E, CDK-4, and CDK-6, and increase in levels of p15 and p21. Both HCT 116 and HT29 lack p16. As seen in Figures $5 \mathrm{a}$ and $\mathrm{b}$ triptolide treatment does not alter levels of these proteins in a way that will explain G1 arrest at doses 100-200 nM. To further confirm that triptolide is not inducing G1 arrest by affecting sequestration of E2F1 by $\mathrm{Rb}$, we evaluated the effect of triptolide on Rb-E2F1 binding by co-immunoprecipitation experiments. As seen in Figures $5 \mathrm{c}$ and $\mathrm{d}$, triptolide treatment does not lead to increased binding of E2F1 to $\mathrm{Rb}$. We next evaluated the effect of triptolide on E2F DNA binding by chromatin immunoprecipitation assay. As seen in Figures $5 \mathrm{e}$ and $\mathrm{f}$, at $12 \mathrm{~h}$ triptolide treatment does not decrease but conversely increases the E2F1 biding to CDC-2 promoter. Our data taken together show that triptolide does not decrease E2F1 DNA binding.

\section{DISCUSSION}

In the current manuscript we demonstrate that triptolide, a diterpene triepoxide from a Chinese herb Trypterigium wilfordii, kills colon cancer cells in vitro. We also demonstrate
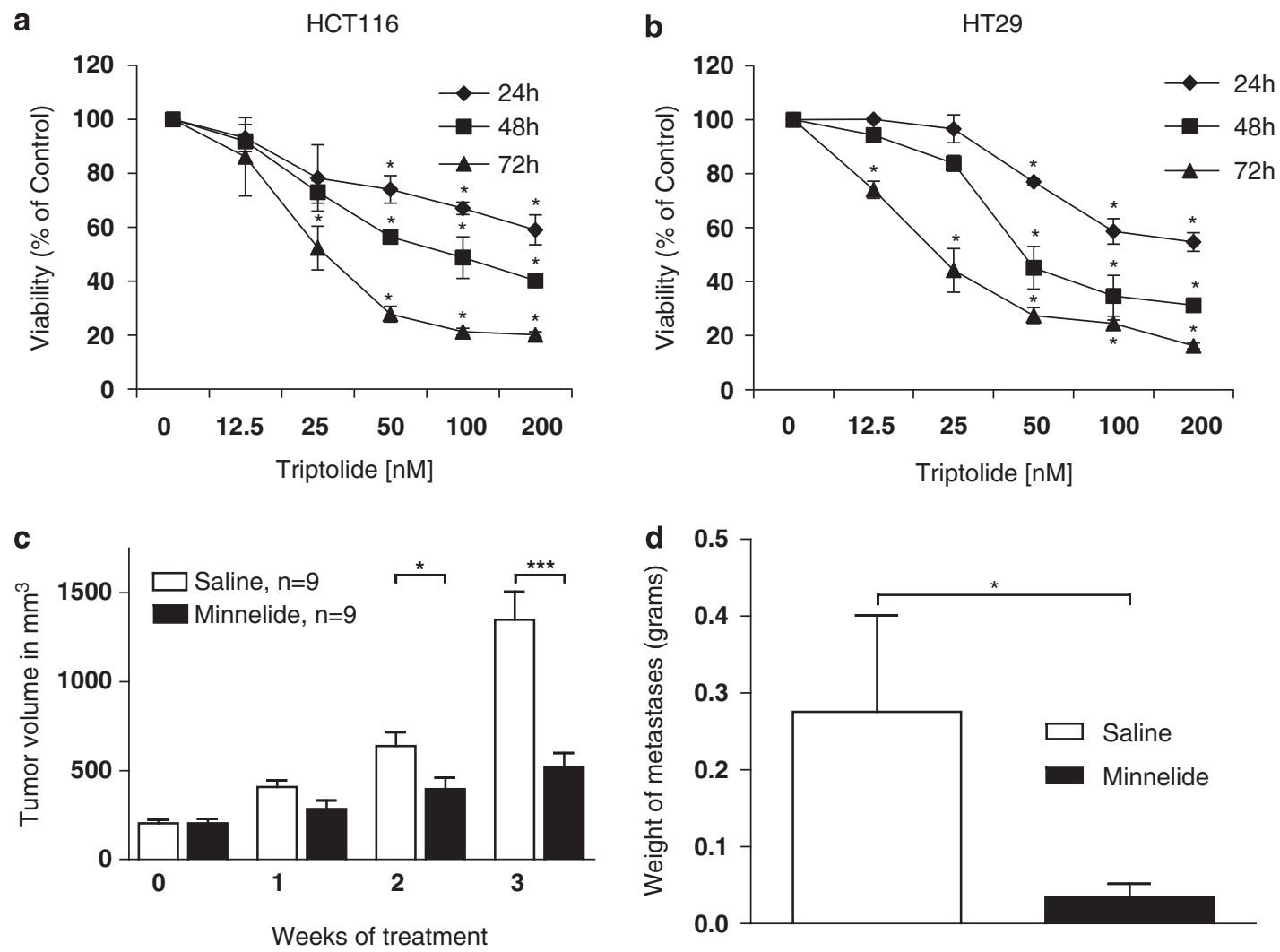

Figure 1 Triptolide and its derivative Minnelide is effective in killing colon cancer cells both in vivo and in vitro: triptolide decreases the viability of colon cancer cell lines (a) HCT116 and (b) HT29 in a dose- and time-dependent manner. (c) In a subcutaneous xenograft model of colon cancer cell line HCT116 treatment with Minnelide leads to decreased growth of tumor when compared with vehicle (saline)-treated animals alone. In a liver metastasis model of colon cancer, Minnelide treatment leads to significantly decreased (d) weight and (e) number of liver metastases. (f) Representative picture of a liver metastases from HCT 116 colon cancer cell line. In the left panel $(10 \times)$ tumor-liver interface is shown. In the right panel, high magnification view of the liver metastases is shown. (g) In a separate liver metastasis model of colon cancer focused on survival, Minnelide treatment significantly improved survival of animals with liver metastases when compared with vehicle treatment alone $(P<0.001)$. (h) Triptolide does not affect the viability of human colonic epithelium cells (HCEC) significantly. 

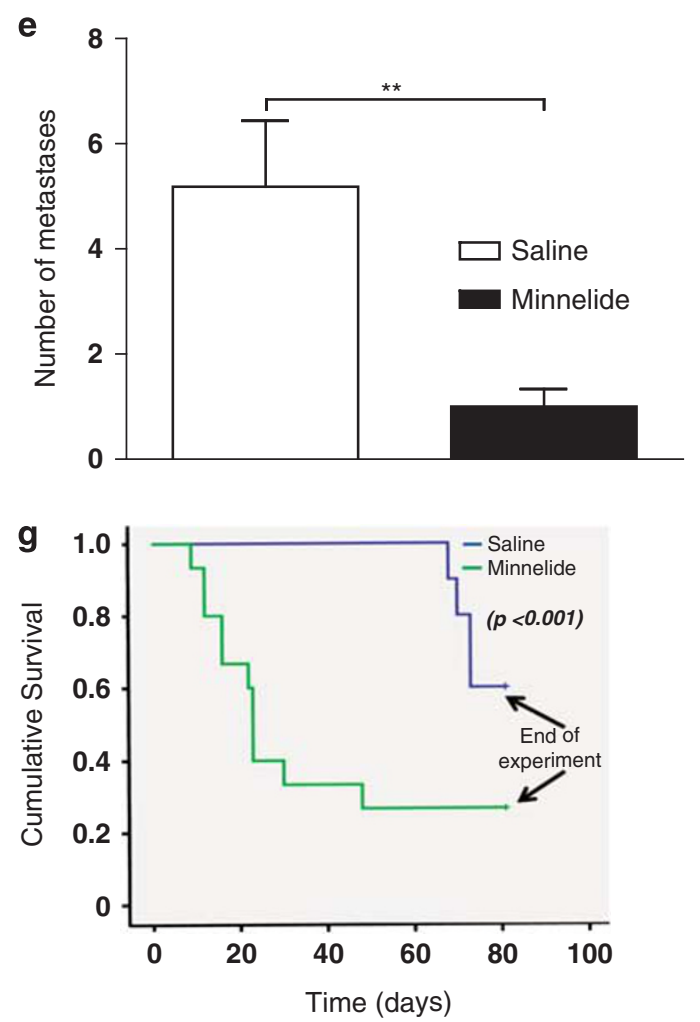

Figure 1 Continued

that Minnelide, a novel water soluble analog of triptolide, is very effective in inhibiting the growth of colon cancer in multiple animal models. Mechanistically, our data suggest that although triptolide activates apoptosis in colon cancer cells, at higher concentrations cell cycle arrest is a more prominent mechanism. Furthermore, we demonstrate that triptolide induces G1-S arrest in colon cancer cells by decreasing E2F1 transcriptional activity at steps downstream of its binding to promoter of E2F1-regulated genes. To our knowledge, this is the first report demonstrating that triptolide can induce G1-S arrest by modulating E2F1 activity. Finally, the evaluation of a novel water soluble analog of triptolide, in a clinically relevant liver metastases model of colon cancer, is an additional strength of the current study.

Triptolide has been evaluated and shown to be effective against multiple cancer types including pancreatic cancer, ${ }^{4}$ neuroblastoma, ${ }^{8}$ osteosarcoma,${ }^{5}$ cholangiocarcinoma, ${ }^{7}$ breast cancer, ${ }^{9}$ glioblastoma, ${ }^{18}$ multiple myeloma, ${ }^{19}$ and prostate cancer. ${ }^{20}$ Triptolide's efficacy has been tested against colon cancer as well. ${ }^{4,11}$ However, detailed evaluation of the mechanism as well as evaluation of a novel water soluble analog in multiple animal models of colon cancer distinguishes the current study from previous work. One of the major obstacles in the application of triptolide in clinical practice has been its low solubility in water. We have recently circumvented this hurdle by synthesis of its water soluble analog Minnelide, ${ }^{12}$ which we have extensively evaluated in pre-clinical models of pancreatic cancer. Phase I clinical trial evaluating Minnelide in patients with pancreatic cancer are currently under way. In the current study we have shown that Minnelide is very effective in both subcutaneous xenograft model as well as liver metastasis model of colon cancer. The utilization of a liver metastasis model of colon cancer is pertinent to the evaluation of the efficacy of any compound against colon cancer, as upwards of $50 \%$ patients with colon cancer develop liver metastases during the course of their disease. ${ }^{21}$ In fact, up to a quarter of patients with colon cancer present with liver metastases at the time of diagnosis. Although liver resection in these patients with colorectal cancer with liver metastases can offer long-term disease-free survival, only a fraction of patients have liver disease amenable to resection. Patients who undergo liver resection are still at risk of recurrent disease. Those who have liver metastases not amenable to resection only have systemic chemotherapy as a treatment option. The fact that Minnelide treatment was able to reduce the number and growth of liver metastases and that it was able to markedly prolong the survival of animals with liver metastases, is very promising. If phase I trial of Minnelide yields encouraging results, it could open doors for evaluation of this novel compound in metastatic as well as primary colon cancer.

The mechanism of action of triptolide or Minnelide is not clearly understood. Our previous studies show that triptolide 
a

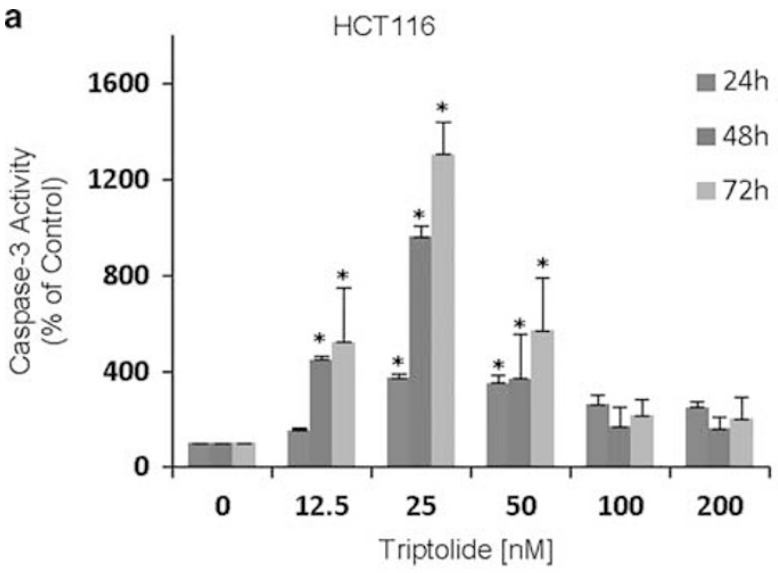

c
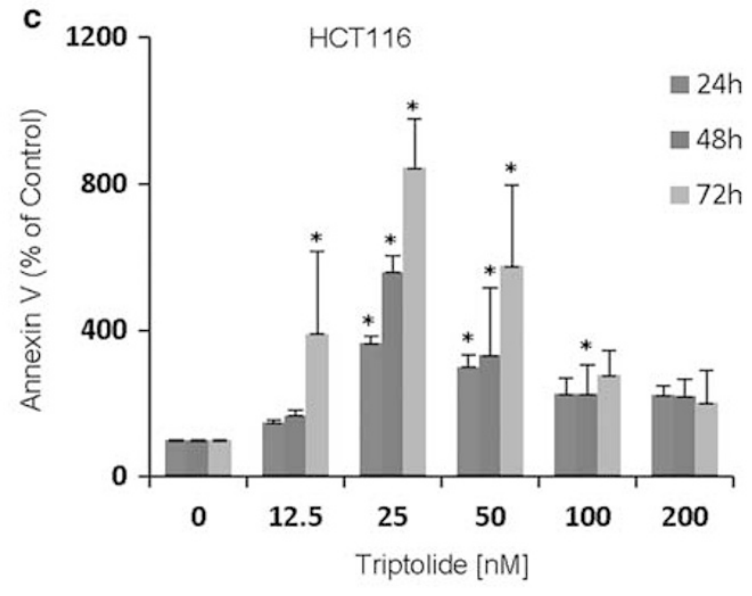

e

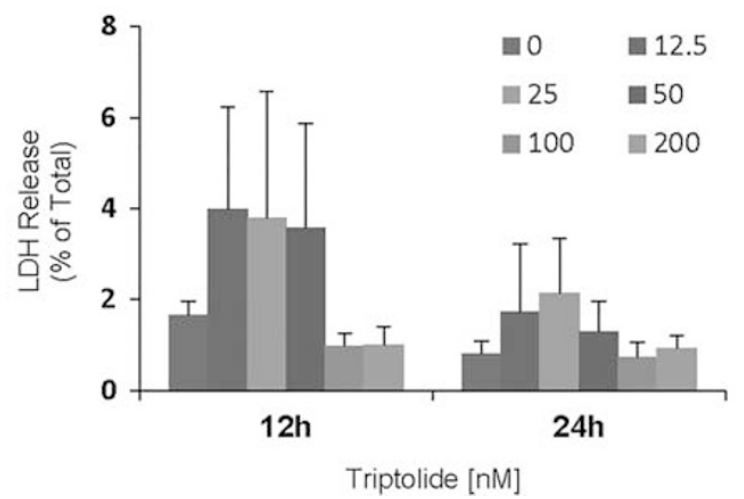

b
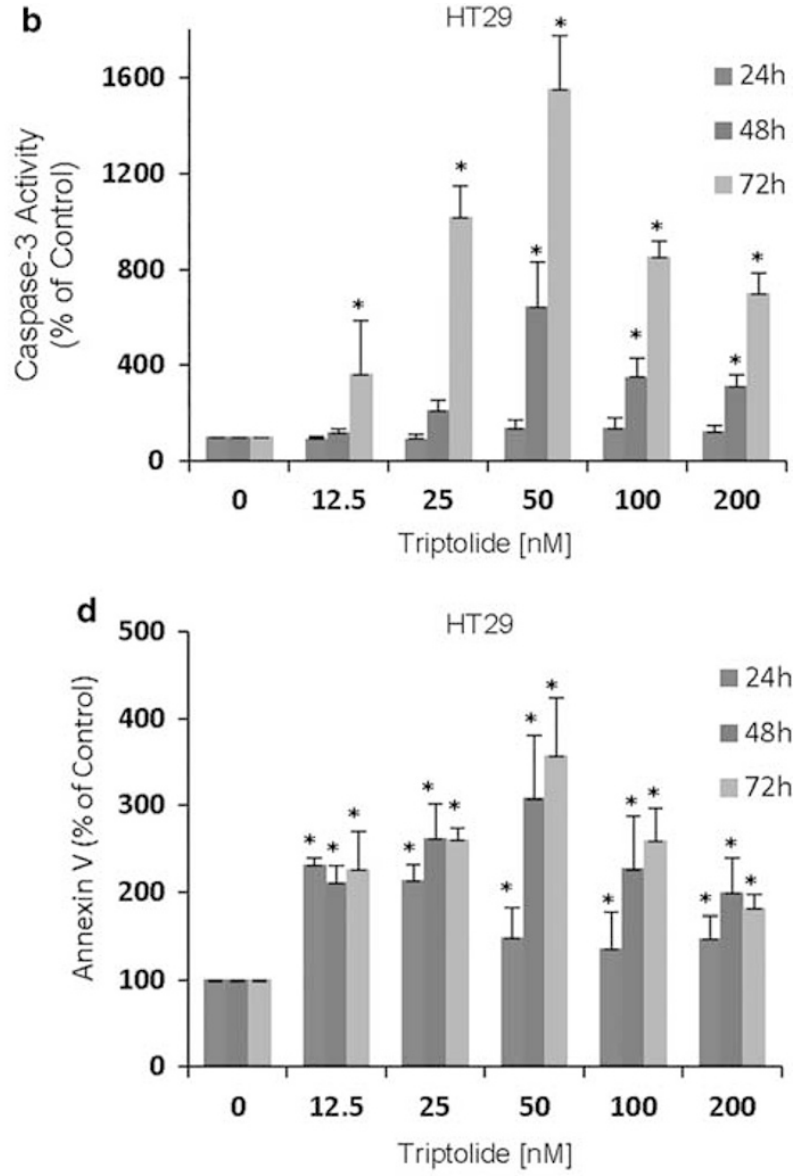

f

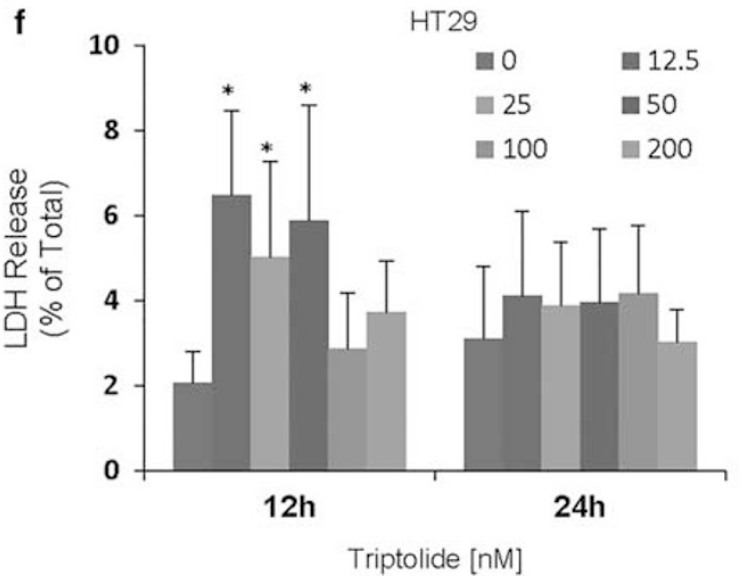

Figure 2 Triptolide treatment leads to activation of apoptosis in colon cancer cells: triptolide treatment leads to caspase-3 activation in both (a) HCT116 and (b) HT29 colon cancer cell lines. However, this activation is not dose-dependent and increases up to 25 nM in HCT116 and 50 nM in HT29 and then decrease at higher concentrations. Similarly, triptolide treatment leads to increased annexin-V staining in both (c) HCT116 and (d) HT29 colon cancer cell lines. As observed with caspase-3 activation, annexin-V staining also is not dose-dependent and increased up to 25 nM in HCT116 and 50 nM in HT29 and then decreased at higher concentrations. Representative flow plot of annexin assay are provided in Supplementary Figure. Triptolide treatment does not lead to significant increase in necrosis, as measured by LDH release in (e) HCT116 colon cancer cells. In (f) HT29 colon cancer cell line, triptolide treatment led to a small but significant increase in LDH release at $12.5-50 \mathrm{~nm}$ dosage at $12-\mathrm{h}$ time point but not at $100-200 \mathrm{~nm}$ dose or with any dose at $24 \mathrm{~h}$.

can induce cell death in cancer cells by multiple mechanisms. We have shown that, depending on the cell type, triptolide can induce either autophagy or apoptosis. ${ }^{15}$ In the current study we demonstrate that triptolide induces apoptosis in colon cancer cells as well. Intriguingly, at higher concentrations the extent of apoptosis decreases as suggested by decreased caspase activation and decreased annexin positivity. However, even with decreasing apoptosis, higher doses of 
a

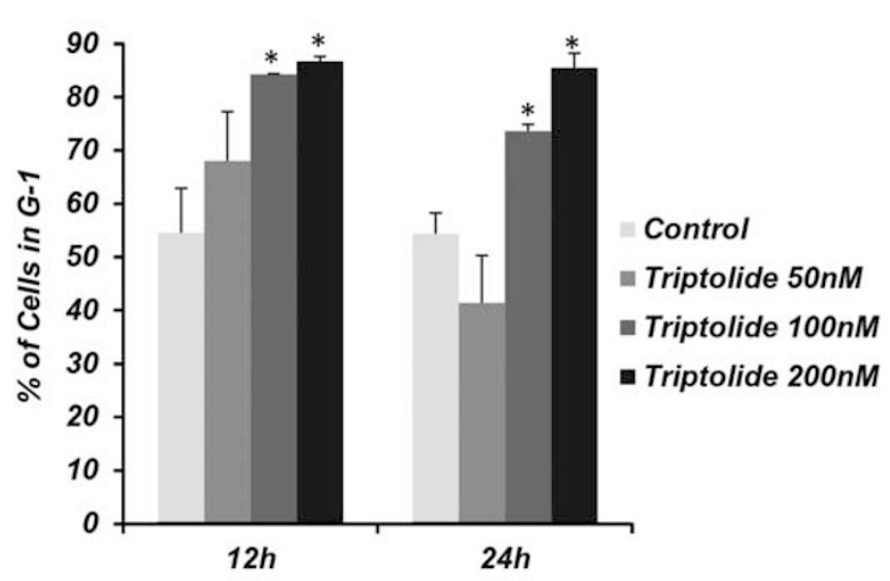

C
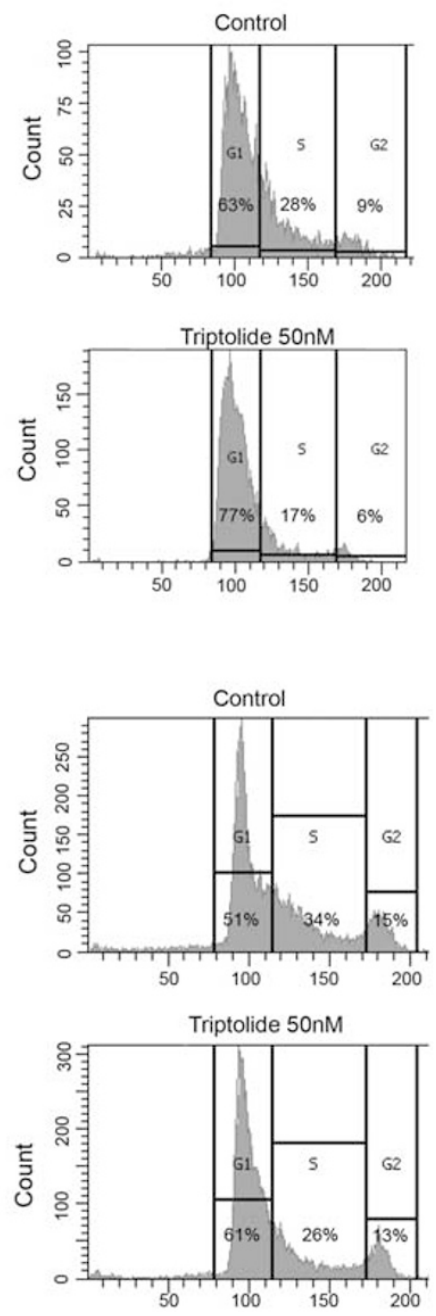

HCT116 12h

HT29 12h b

HT29
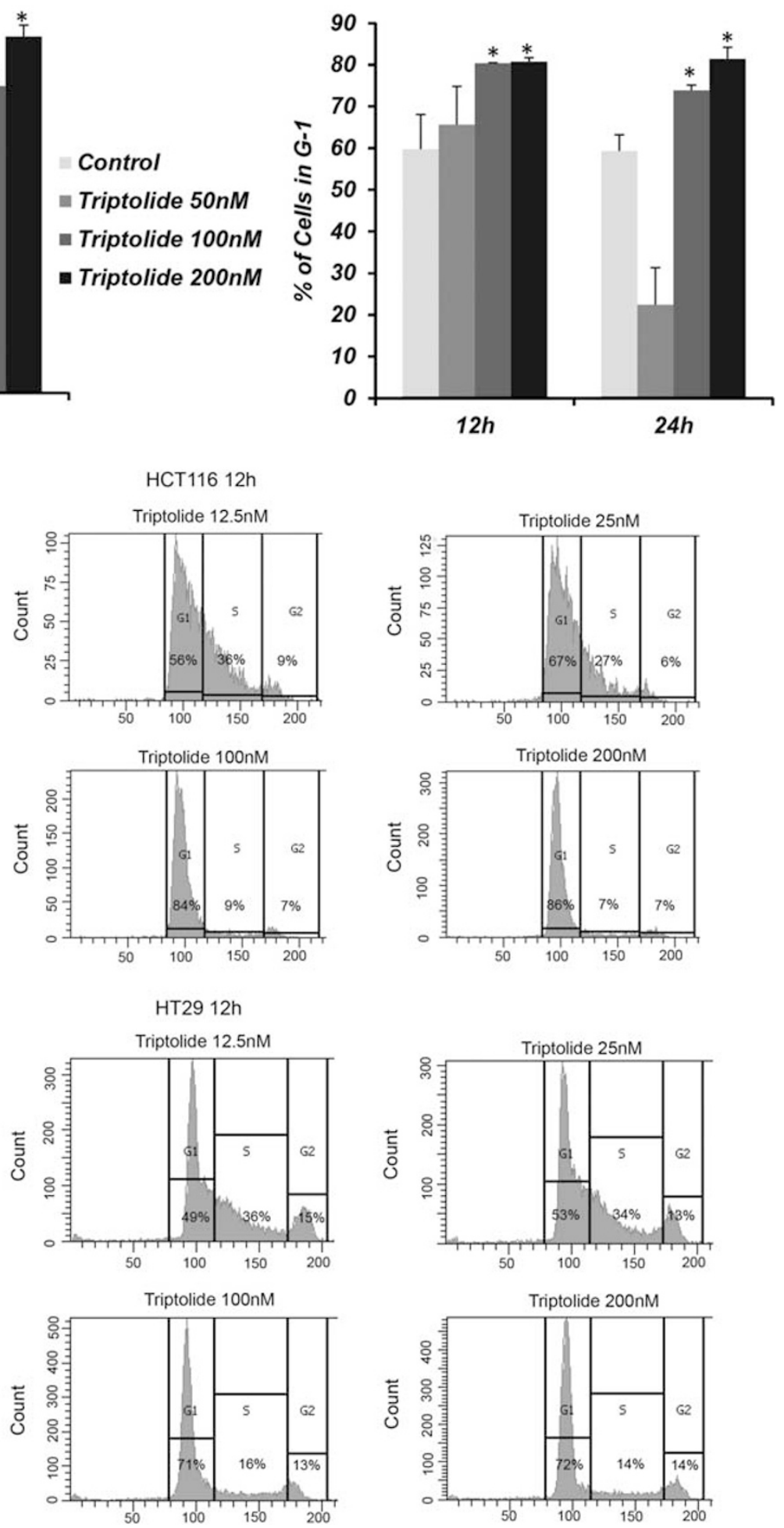

Figure 3 Triptolide treatment leads to G1-S arrest. Triptolide treatment leads to significantly increased proportion of cells in G1 fraction at a concentrations of 100-200 nM when compared with untreated controls in both (a) HCT116 and (b) HT29 cells. (c) A representative run of cell cycle analysis for both cell lines is shown. 
a

HCT116
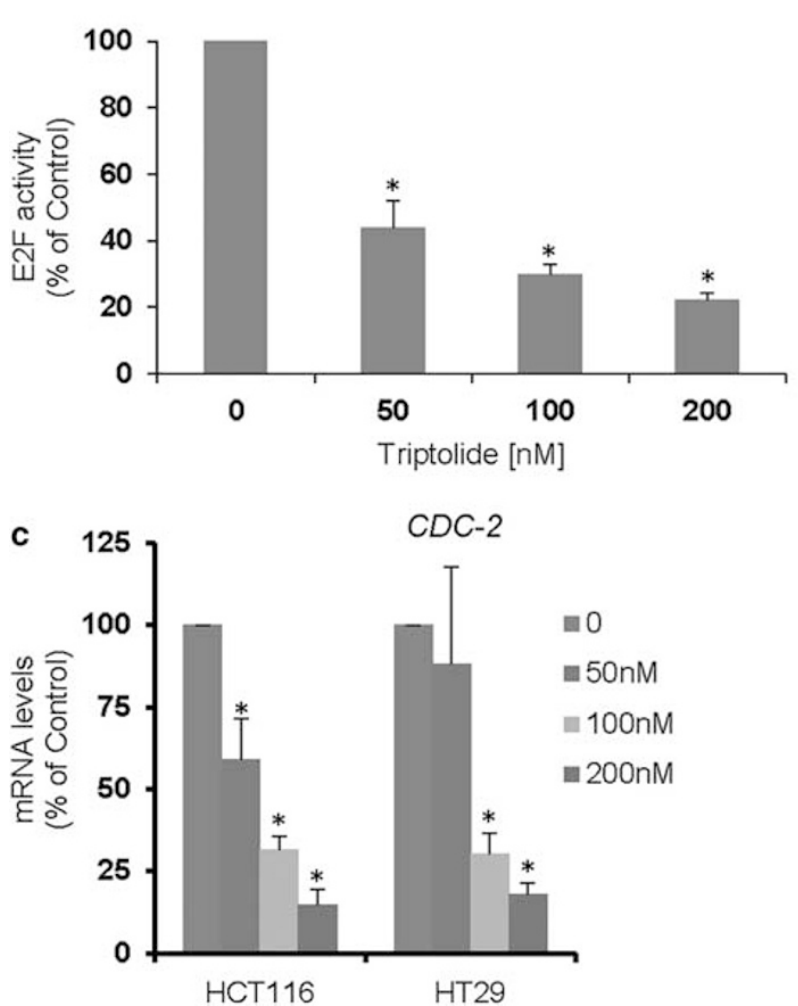
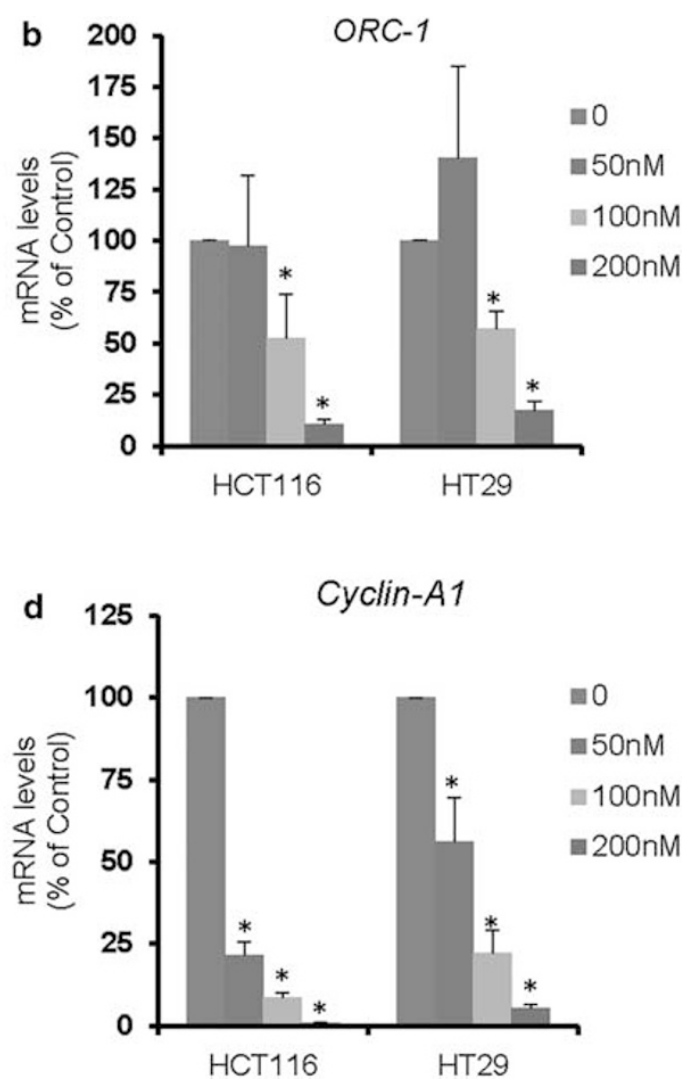

Figure 4 Triptolide inhibits transcriptional activity of E2F. (a) Triptolide treatment leads to decreased E2F transcriptional activity as measured by luciferase promoter binding assay in HCT116 cells. Triptolide treatment also leads to decrease in the mRNA levels of genes known to be transcriptionally regulated by E2F1, including (b) ORC-1, (c) CDC-2, and (d) cyclin A-1, in both HCT116 and HT29 cells.

triptolide were able to decrease the viability of colon cancer cell lines, suggesting that another mechanism of cell death was functional at higher doses. Our data suggests that higher concentrations of triptolide did not induce autophagy or necrosis but did induce G1 cell cycle arrest. Triptolideinduced cell cycle arrest has been seen in other cancer cell types including colon cancer. ${ }^{11,18,19}$ Zhao et al ${ }^{19}$ have shown that triptolide decreases proliferation of multiple myeloma cells and induces G0-G1 cell cycle arrest. Zhang et al ${ }^{18}$ demonstrated that triptolide inhibits proliferation and invasion potential of malignant glioma cell lines by inducing G0/ G1 arrest. Similarly Liu et al ${ }^{11}$ have observed that triptolide induces G0-G1 cell cycle arrest without inducing apoptosis in colon cancer cell lines. That triptolide induces a biphasic response, predominant apoptosis at low concentrations and cell cycle arrest at high concentrations, has not been described before.

Various disease or cell type-specific mechanisms of triptolide-induced G0/G1 arrest have been proposed. G1 to $\mathrm{S}$ transition is controlled by $\mathrm{Rb}$, a member of the family of pocket proteins, and is mediated by its binding to E2F1. The ability of $\mathrm{Rb}$ to bind to E2F1 and modulate its activity is in itself regulated by its cell cycle-dependent phosphorylation. Hypophosphorylated form of $\mathrm{Rb}$ predominates during $\mathrm{G} 0$ and early G1 and binds to E2F. Increasing degree of Rb phosphorylation initially by CDK-4 and CDK-6 along with cyclin D and subsequently by CDK-2 in combination with cyclin E causes E2F release and G1-S progression. ${ }^{22}$ $\mathrm{Rb}$ phosphorylation by cyclin/CDK complexes is further regulated by CDK inhibitors, the Ink4 protein family ( $\mathrm{p} 15$, p16, p18, p19) and Cip/Kip protein family (p21, p27, and p57). Cellular stresses as well as other inhibitors like transforming growth factor $\beta$ can stall cell cycle progression through inhibition of cyclin/CDK complexes by CDK inhibitors and maintenance of $\mathrm{pRb}$ in the hypophosphorylated form. ${ }^{23}$ Modulation of any of these events/proteins can lead to G1-S arrest. Previously published literature suggests that triptolide could induce cell cycle arrest by modulating many of these proteins. Zhang et al ${ }^{18}$ have shown that triptolide induces G1-S arrest in glioblastoma cells by decreasing levels of cyclin D1, CDK-4, and CDK-6 and by decreased phosphorylation of $\mathrm{Rb}$. In prostate cancer, Li et a ${ }^{20}$ have demonstrated that that triptolide interferes with G1/S phase transition leading to a loss of cell viability in two cell lines independent of their p53 status. Recently, a study by Liu et $a l^{11}$ in colon cancer cell lines HT29 and SW480 demonstrated that triptolide-induced G1 arrest in these cell lines by increasing levels of $\mathrm{p} 21$, which then inhibits CDK-4 
a

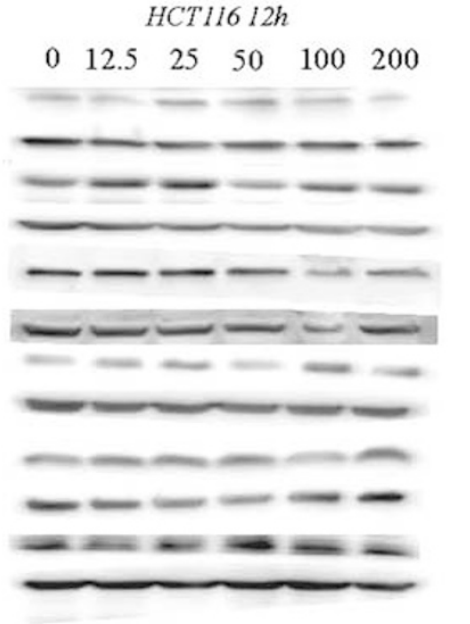

C
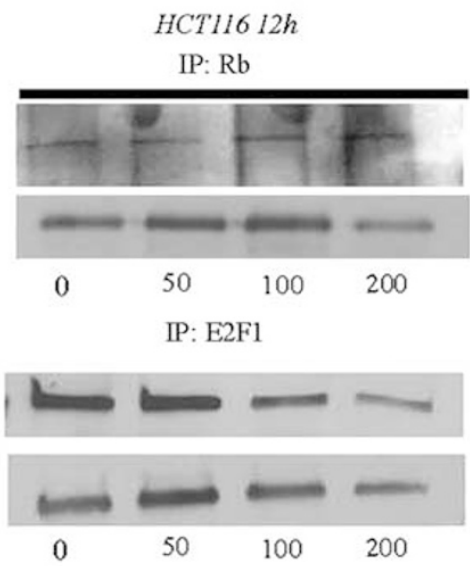

e

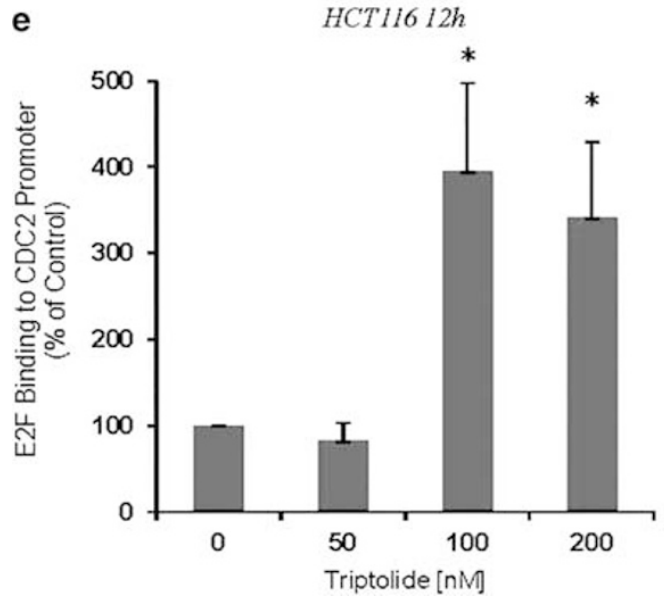

Triptolide [nM]

Cyclin D1

Actin

Cyclin E2

Actin

CDK4

Actin

CDK6

Actin

p15

Actin

p21

Actin

$\mathrm{Rb}$

E2F1

Triptolide [nM]

$\mathrm{Rb}$

E2F1

Triptolide [nM] b

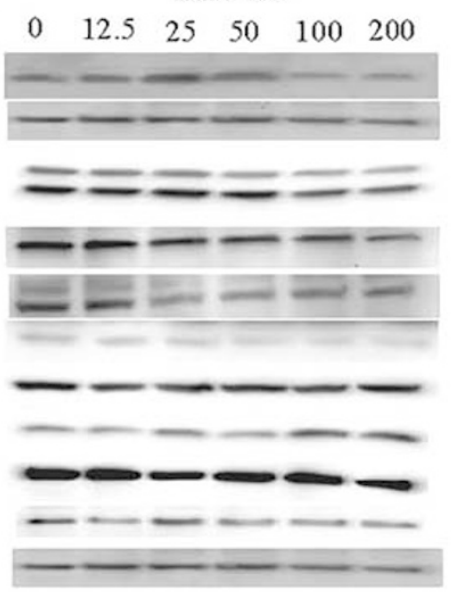

d

HT29 12h
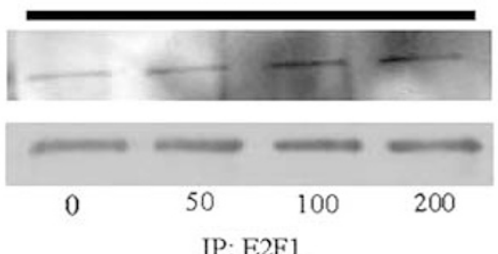

IP: E2F1

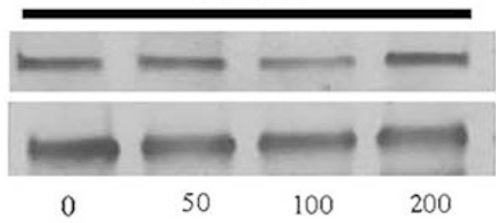

HT29 $12 \mathrm{~h}$

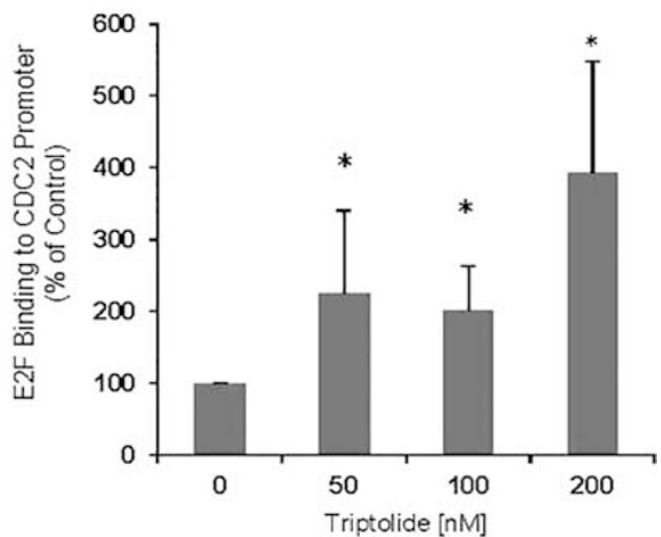

Figure 5 Triptolide does not modulate E2F1-Rb binding. Triptolide treatment does not decrease the level of cyclin D or cyclin E, CDK4 or CDK6 or p15 or p21, proteins which regulate E2F1-Rb binding, in either (a) HCT116 or (b) HT29 colon cancer cells. The full-length blots demonstrating the protein of interest and actin are shown in Supplementary Figure 3 and 4. Triptolide does not modulate binding of E2F1 and Rb as measured by co-immunoprecipitation in either (c) HCT116 or (d) HT29 cancer cells. E2F1 or Rb was immunoprecipitated from colon cancer cells lysates treated with triptolide and the immunoprecipitates were separated on SDS page and probed for both Rb and E2F1. Triptolide treatment for $12 \mathrm{~h}$ increases binding of E2F1 to promoter region of E2F1 responsive genes (CDC-2) in both (e) HCT116 and (f) HT29 colon cancer cells. 
and CDK-6. This is intriguing as HT29 is a p53-mutated cell line and p21 is a p53-controlled gene. Moreover, in this study the concentration of triptolide, which induces cell cycle arrest was lower ( $50 \mathrm{~nm}$ and lower) than what we observed in our study (50 nM and higher).

In contrast, our data suggest that triptolide induces cell cycle arrest in HCT116 (wild type p53) and HT29 (p53 mutant) by inhibiting E2F transactivation. Our data also suggest that triptolide does not modulate Rb-E2F1 binding and does not decrease cyclin D or cyclin E, CDK-4 or CDK-6, or increase p21 levels. Results from chromatin immunoprecipitation assay demonstrate that triptolide-mediated inhibition of E2F1 activity is not through inhibition of E2F1 binding to promoter sites. These together suggest that the inhibition of E2F1 transactivation must happen by its effect on events beyond E2F1 DNA binding. Events that regulate E2F1 activity beyond its binding to DNA are not well known. Similar to many other transcription factors, E2F1 transcriptional activity has been shown to be regulated by acetylation. ${ }^{24}$ Also, triptolide has been shown to regulate the transcriptional activity of many other transcription factors like $\mathrm{NFKB}^{25}$ and HSF- ${ }^{26}$ by influencing post-DNA-binding events. Elucidation of the post E2F1 DNA-binding events, which are modulated by triptolide would lead to progress in our understanding of mechanism of action of triptolide.

The fact that triptolide leads to apoptosis at low doses and cell cycle arrest at high doses is also very intriguing. Cell cycle arrest and apoptosis are closely linked events and many of the events in apoptosis are regulated by genes involved in cell cycle progression. ${ }^{27}$ For example, c-Myc can induce both proliferation and apoptosis and cell fate may depend on the presence or absence of mitogens. ${ }^{27}$ Similarly, p53 activation can induce cell cycle arrest or cell death depending on many factors including p21 status of cell, crosstalk with pRb, and extent of DNA damage. Interestingly, data suggest that E2F besides regulating cell cycle regulates apoptotic pathways as well. Few of the apoptotic E2F1 target genes include apoptosis protease-activating factor-1, p73, and ARF. ${ }^{28,29}$ E2F1 can induce apoptosis in both p53-dependent and -independent fashion. ${ }^{22}$ It is interesting that in the current study triptolide decreased E2F1 activity and at the same time a reduction in apoptotic parameters was observed. Though not conclusive, this may suggest that E2F1 inhibition may contribute to cell cycle arrest and inhibition of apoptosis observed with high doses of triptolide.

In summary, our data demonstrates that triptolide and its novel water soluble analog Minnelide are very effective in killing colon cancer cells in vitro and in reducing growth of primary colon tumors as well as colorectal metastases to the liver in vivo, respectively. We also show that triptolide leads to predominant apoptosis at low dose and cell cycle arrest at high dose. This is first report that reveals biphasic effect of triptolide on apoptosis and cell cycle. Our results also suggest that triptolide induces cell cycle arrest by inhibiting E2F1 activity by inhibiting its transcriptional activity. Overall
Minnelide could emerge as novel therapeutic strategy for primary as well as metastatic colon cancer.

Supplementary Information accompanies the paper on the Laboratory Investigation website (http://www.laboratoryinvestigation.org)

\section{ACKNOWLEDGMENTS}

We also acknowledge the grant support from Minnesota Partnership for Biotechnology and Medical Genomics.

\section{DISCLOSURE/CONFLICT OF INTEREST}

$\mathrm{R}$ Chugh has ownership interest in a patent of Minnelide. AK Saluja has ownership interest (including patents) in Minneamrita therapeutics and is a consultant/advisory board member for Minneamrita Therapeutics. The remaining authors declare no conflict of interest.

1. Siegel R, Naishadham D, Jemal A. Cancer statistics, 2013. CA Cancer J Clin 2013;63:11-30.

2. Andre T, Boni C, Navarro $M$ et al. Improved overall survival with oxaliplatin, fluorouracil, and leucovorin as adjuvant treatment in stage II or III colon cancer in the MOSAIC trial. J Clin Oncol 2009;27: 3109-3116.

3. Braun $\mathrm{AH}$, Achterrath $\mathrm{W}$, Wilke $\mathrm{H}$. New systemic frontline treatment for metastatic colorectal carcinoma. Cancer 2004;100:1558-1577.

4. Phillips PA, Dudeja V, McCarroll JA et al. Triptolide induces pancreatic cancer cell death via inhibition of heat shock protein 70. Cancer Res 2007;67:9407-9416.

5. Banerjee S, Thayanithy V, Sangwan V et al. Minnelide reduces tumor burden in preclinical models of osteosarcoma. Cancer Lett 2013;335: 412-420.

6. Rousalova I, Banerjee S, Sangwan V et al. Minnelide: a novel therapeutic that promotes apoptosis in non-small cell lung carcinoma in vivo. PLoS One 2013;8:e77411.

7. Clawson KA, Borja-Cacho D, Antonoff MB et al. Triptolide and TRAIL combination enhances apoptosis in cholangiocarcinoma. J Surg Res 2010;163:244-249.

8. Antonoff MB, Chugh R, Borja-Cacho D et al. Triptolide therapy for neuroblastoma decreases cell viability in vitro and inhibits tumor growth in vivo. Surgery 2009;146:282-290.

9. Yang S, Chen J, Guo Z et al. Triptolide inhibits the growth and metastasis of solid tumors. Mol Cancer Ther 2003;2:65-72.

10. Fidler JM, Li K, Chung C et al. PG490-88, a derivative of triptolide, causes tumor regression and sensitizes tumors to chemotherapy. Mol Cancer Ther 2003;2:855-862.

11. Liu J, Shen M, Yue Z et al. Triptolide inhibits colon-rectal cancer cells proliferation by induction of $\mathrm{G} 1$ phase arrest through upregulation of p21. Phytomedicine 2012;19:756-762.

12. Chugh R, Sangwan V, Patil SP et al. A preclinical evaluation of Minnelide as a therapeutic agent against pancreatic cancer. Sci Transl Med 2012;4156ra39.

13. Chen Z, Sangwan V, Banerjee S et al. miR-204 mediated loss of Myeloid cell leukemia-1 results in pancreatic cancer cell death. Mol Cancer 2013;12:105

14. Banerjee S, Sangwan V, McGinn O et al. Triptolide-induced cell death in pancreatic cancer is mediated by O-GlcNAc modification of transcription Factor Sp1. J Biol Chem 2013;288:33927-33938.

15. Mujumdar N, Mackenzie TN, Dudeja V et al. Triptolide induces cell death in pancreatic cancer cells by apoptotic and autophagic pathways. Gastroenterology 2010;139:598-608.

16. Roig Al, Eskiocak U, Hight SK et al. Immortalized epithelial cells derived from human colon biopsies express stem cell markers and differentiate in vitro. Gastroenterology 2010;138:1012-1021.

17. Dudeja V, Chugh RK, Sangwan V et al. Pro-survival role of heat shock factor 1 (HSF1) in the pathogenesis of pancreatobiliary tumors. Am J Physiol Gastrointest Liver Physiol 2011;300:G948-G955.

18. Zhang $H$, Zhu W, Su $X$ et al. Triptolide inhibits proliferation and invasion of malignant glioma cells. J Neurooncol 2012;109:53-62. 
19. Zhao F, Chen Y, Li R et al. Triptolide alters histone H3K9 and H3K27 methylation state and induces G0/G1 arrest and caspase-dependent apoptosis in multiple myeloma in vitro. Toxicology 2010;267:70-79.

20. Li W, Liu Y, Li XX et al. MAPKs are not involved in triptolide-induced cel growth inhibition and apoptosis in prostate cancer cell lines with different p53 status. Planta Med 2011;77:27-31.

21. Wanebo HJ, LeGolvan M, Paty PB et al. Meeting the biologic challenge of colorectal metastases. Clin Exp Metastasis 2012;29:821-839.

22. Stevens C, La Thangue NB. E2F and cell cycle control: a doubleedged sword. Arch Biochem Biophys 2003;412:157-169.

23. Sherr CJ, Roberts JM. CDK inhibitors: positive and negative regulators of G1-phase progression. Genes Dev 1999;13:1501-1512.

24. Martinez-Balbas MA, Bauer UM, Nielsen SJ et al. Regulation of E2F1 activity by acetylation. Embo J 2000;19:662-671.
25. Zhu W, Ou Y, Li Y et al. A small-molecule triptolide suppresses angiogenesis and invasion of human anaplastic thyroid carcinoma cells via down-regulation of the nuclear factor-kappa B pathway. Mol Pharmacol 2009;75:812-819.

26. Westerheide SD, Kawahara TL, Orton $\mathrm{K}$ et al. Triptolide, an inhibitor of the human heat shock response that enhances stress-induced cell death. J Biol Chem. 2006;281:9616-9622.

27. Pucci B, Kasten M, Giordano A. Cell cycle and apoptosis. Neoplasia 2000;2:291-299.

28. Irwin M, Marin MC, Phillips AC et al. Role for the $\mathrm{p} 53$ homologue $\mathrm{p} 73$ in E2F-1-induced apoptosis. Nature 2000;407:645-648.

29. Moroni MC, Hickman ES, Lazzerini Denchi E et al. Apaf-1 is a transcriptional target for E2F and p53. Nat Cell Biol 2001;3: 552-558. 